\title{
Estrogen nuclear receptors affect cell migration by altering sublocalization of AQP2 in glioma cell lines
}

\author{
Shu Wan ${ }^{1}$, Juanjuan Jiang ${ }^{2}$, Chuanming Zheng ${ }^{3}$, Ning Wang ${ }^{4}$, Xia Zhai ${ }^{5}$, Xiangwei Fei ${ }^{4}$, Ruijin Wu Wu $^{4}$ Xiuxiu Jiang ${ }^{4}$
}

\begin{abstract}
Glioblastomas are capable of infiltrating into neighboring brain tissues. The prognosis of a male patient is worse than that of women. Here, we demonstrate the effects of estrogen on invasion of glioma cells via regulating estrogen nuclear receptors (ERa and ERß) combined with aquaporin 2 (AQP2). In our study, we conclude that AQP2 was located mainly in the nuclei of the glioma cell lines and is capable of inhibiting cell invasion. According to the gene ontology analysis, out of 138 screened genes, three genes of ankyrin repeat and FYVE domain containing 1 (ANKFY1), lymphocyte transmembrane adaptor 1 (LAX1), and latent transforming growth factor beta-binding protein 1 (LTBP1) were found to be regulating the ERa and ER $\beta$. The expression of ERa was found to be high, whereas the expression of both ER $\beta$ and AQP2 was low in glioma cells from patient tissues and glioblastoma cell lines. The expression levels of $A Q P 2, A N K F Y 1, L A X 1$, and LTBP1 were upregulated by both ERa small interfering RNA (siRNA) and overexpression of ERß. AQP2 inhibition of cell invasion was inversely influenced by LAX1 siRNA. The luciferase report system indicated that AQP2 promoted the transcriptional activity of LAX1 and inhibited cell invasion. These data suggest that ER $\beta$ may function as AQP promoter in the nucleus to sustain cells' stability by promoting AQP production, while ERa acts as an antagonist of AQP2. The ratio between ERa and ERB is likely to affect the distribution of AQP2 in the nucleus. Low level of ER $\beta$ reduces the inhibition of invasion of glioma cells influenced by high level of LAX1 expression, leading to an increase in the invasion ability of glioma cells.
\end{abstract}

\section{Introduction}

Gliomas are the most common type of primary malignant brain tumors, having an overall poor prognosis. Evidence from a number of sources has suggested that female sex hormones play an important role in the development of gliomas in women ${ }^{1}$. Astrocytes express receptors for gonadal hormones and produce several neurosteroids, including estradiol (E2), progesterone, and testosterone ${ }^{2}$. Recent reports and clinical trials depicted

\footnotetext{
Correspondence: Xiuxiu Jiang (jiangxiuxiu0418@zju.edu.cn)

${ }^{1}$ Department of Neurosurgery, The First Affiliated Hospital, Zhejiang University School of Medicine, Hangzhou Shi, Zhejiang Province, China

2Department of Otolaryngology, Hangzhou Children's Hospital, Hangzhou City, Zhejiang Province, China

Full list of author information is available at the end of the article

These authors contributed equally: Shu Wan, Juanjuan Jiang, Chuanming Zheng

Edited by M.V. Niklison Chirou
}

the roles of estrogen receptor (ER) agonists in protecting the central nervous system from noxious consequences of neuroinflammation and neurodegeneration ${ }^{3}$. However, the role of estradiol in glioma migration and proliferation remains controversial. Malignant gliomas can infiltrate and spread widely into neighboring brain tissues. Sex steroids have been implicated in the development and progression of primary brain tumor ${ }^{4}$. For instance, androgen promotes glioma cell proliferation ${ }^{5}$, while progesterone promotes proliferation and invasion of glioma cells ${ }^{6}$. It is generally accepted that estrogen functions as a tumor promoter that induces cytoprotective mechanisms, which reduce apoptosis in medulloblastomas ${ }^{7}$. On the other hand, estrogens have a neuroprotective role in several neurological disorders, such as Parkinson's

\section{(c) 2018 The Author(s).}

\footnotetext{
(c) (i) Open Access This article is licensed under a Creative Commons Attribution 4.0 International License, which permits use, sharing, adaptation, distribution and reproduction cc) in any medium or format, as long as you give appropriate credit to the original author(s) and the source, provide a link to the Creative Commons license, and indicate if changes were made. The images or other third party material in this article are included in the article's Creative Commons license, unless indicated otherwise in a credit line to the material. If material is not included in the article's Creative Commons license and your intended use is not permitted by statutory regulation or exceeds the permitted use, you will need to obtain permission directly from the copyright holder. To view a copy of this license, visit http://creativecommons.org/licenses/by/4.0/.
} 
disease, Alzheimer's disease, and cerebrovascular accidents ${ }^{8}$. These neuroprotective effects include increased cell proliferation, cell migration, and inflammation. Cell migration is a highly orchestrated process. Estrogens promote neuronal differentiation, migration, and survival in the brain. Dysregulation of cell migration develops into tumor growth and metastatic processes. Due to intensive research findings regarding the role of E2 in promoting gliomas, the molecular mechanism of estrogen signaling cannot be ignored.

Aquaporins (AQPs) are small membrane channel proteins that control water and small solute transport through the phospholipid bilayers. AQPs are subdivided into water-selective channels and aquaglyceroporins ${ }^{9}$. In mammals, 13 isoforms (AQP0-AQP12) of the AQP protein family have been identified that have different properties of cellular localization and permeability for water. A growing body of evidence has suggested that AQPs could facilitate cell migration, invasion, and proliferation in tumor development with the aid of water transport ${ }^{10,11}$. Studies of tumor angiogenesis have revealed an unexpected function of AQPs in cell migration, invasion, and the spread of malignancy ${ }^{12,13}$. For instance, AQP1 increases cell migration and the metastatic potential of melanoma cells ${ }^{14}$. AQP4 is upregulated in astrocytomas and metastatic tumors, and the depletion of AQP4 reduces astrocyte cell migration $^{15,16}$. In the reproductive system, AQP1, AQP5, and AQP8 have been shown to mediate E2-induced cell migration ${ }^{17}$. Our previous study also showed that AQP2 mediates E2-induced cell migration, invasion, and adhesion in endometrial cancer ${ }^{18}$. AQP2 was originally found in the collecting ducts of the kidneys, and it functions as a vasopressin-sensitive water channel in principal cells ${ }^{19}$. Our previous studies demonstrated that AQP2 was also expressed in the human endometrium and endometriosis. E2 might regulate the expression of $\mathrm{AQP} 2$ in endometrial cells $^{20,21}$. However, the mechanism by which AQP2 mediates nervous system tumoral processes is still unclear. There is a lack of direct mechanistic evidence to explain AQP2 regulation of cell migration and invasion of glioma cells.

Therefore, it is indispensable to clarify the signaling responses triggered by $\mathrm{E}_{2}$ that are relevant to antiglioma effects. We aimed to investigate AQP2 involvement in estrogen-dependent glioma invasion to gain relevant mechanistic insights into this process. The experimental results from the present study provide several novel pieces of information regarding the mechanisms of AQP2 function and sublocalization in glioma by E2 and ERs. This work also provides us with the possibility for plausible antitumor therapy by targeting water channels in gliomas.

\section{Results}

Expression levels of AQP2 and ERs in human glioma cells

Based on the fluorescent staining (FS) results, we assume that AQP2 is located mainly in the outer part of the nuclei in glial and glioma cells in the tissues (Fig. 1a). Western blot analysis showed that expression levels of AQP2 were decreased in glioma cells compared to that in glial cells from the tissues (Fig. 1b). The expression levels of AQP2 in glioma cells from low-grade tumors (i.e., stages I and II) were lower in glioma cells than those in high-grade tumors (stages III and IV), but there was no statistical difference (Fig. 1b, c). It is generally accepted that ER $\alpha$ functions as a tumor promoter ${ }^{22}$, while ER $\beta$ functions as a tumor suppressor ${ }^{23}$. There was no report of the presence of GPR30 in the gliomas. In this study, we tested several ERs, including ER $\alpha, E R \beta$, and GPR30 in glioma and glial cells (Fig. 1b, c). ER $\alpha$ protein expression was higher in glioma cells than in glial cells. ER $\beta$ was mainly expressed in the astrocytes of low-grade gliomas and in normal astrocytes. ER $\beta$ levels were significantly decreased in glioma cells compared to those in glial cells. There was no significant difference in the expression of GPR30 between glioma and glial cells.

\section{E2 promotes cell migration and invasion via AQP2 in U87 cells}

To investigate the effects of E2 on cell migration and invasion, the T98G and U87 cell lines were treated with different concentrations $\left(10^{-9}, 10^{-8}\right.$, or $\left.10^{-7} \mathrm{M}\right)$ of $\mathrm{E} 2$ dissolved by dimethyl sulfoxide for $24 \mathrm{~h}$. Based on the results, it is proven that the relationship between E2 and both cell lines on invasion and migration is directly proportional, among which cell migration and cell invasion occur in an E2 concentration-dependent manner (Fig. 2a-d). To investigate the relationship between E2 and AQP2, notably, the protein (Fig. 2e) and mRNA levels of AQP2 were downregulated by E2 by $40-60 \%$ (Fig. 2f). Overexpression of AQP2 with the AQP2 vector (pSLLVCMV-Zsgreen-puro) significantly decreased basal glioma cell invasion. Overexpression of AQP2 attenuated the stimulatory effect on cell invasion induced by E2 $\left(10^{-7} \mathrm{M}\right)$ (Fig. 2g, h). To investigate whether overexpression of AQP2 and AQP2 + E2 induced cell apoptosis, we conducted a cell proliferation experiment using CCK-8 solution and the result showed E2-promoted cell proliferation at a time-dependent but not concentrationdependent manner, while AQP2 had no effect on cell proliferation. These data meant that the decreased cell number in Fig. $2 \mathrm{~g}$ was due to decreased cell migration number.

\section{E2 decreases intranuclear AQP2}

To further investigate the sublocalization of AQP2 in glioblastoma cell lines, we performed FS in glioblastoma 
A
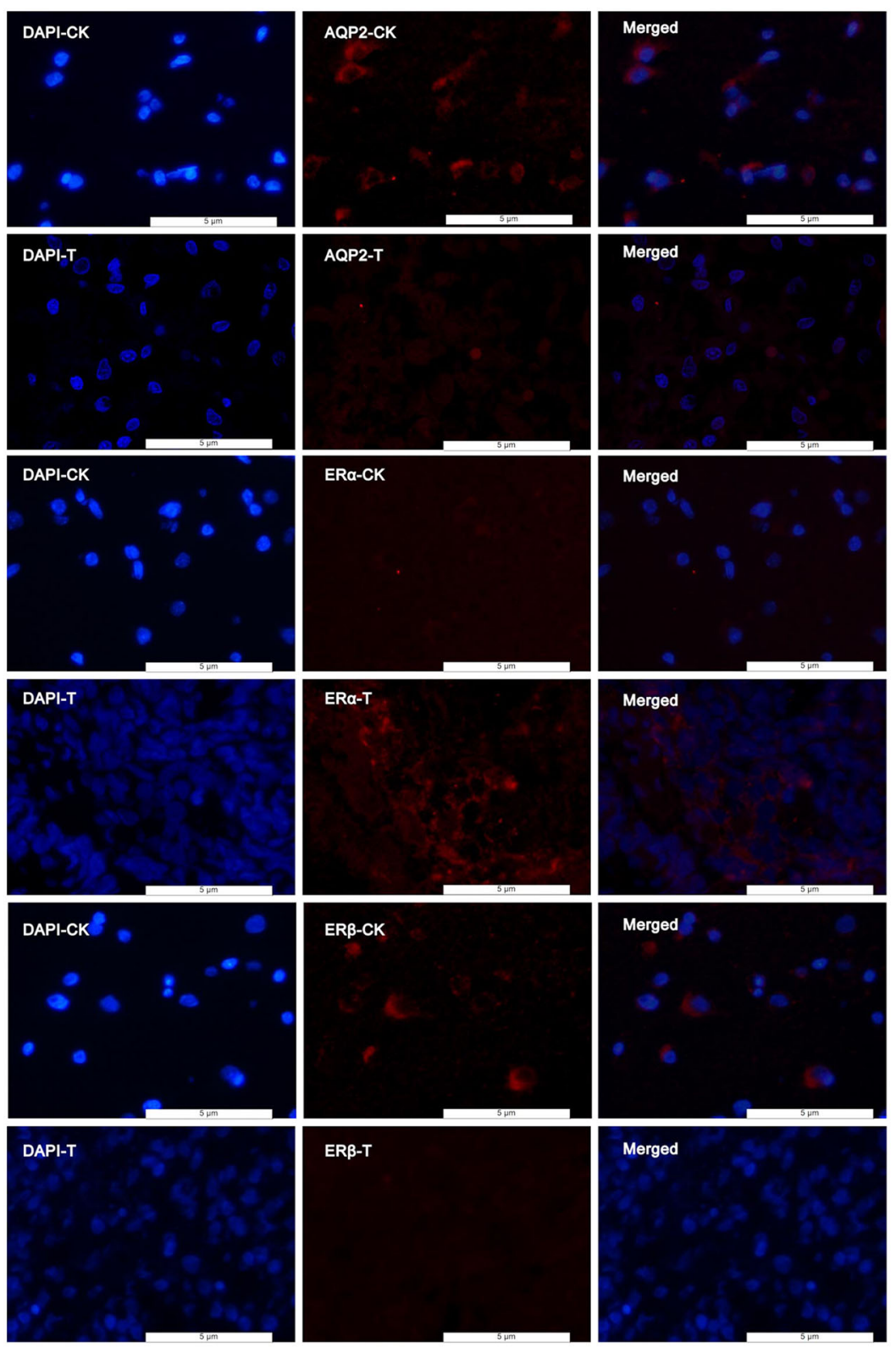

B

CK CK CK CK TL1 TL2 TL3 TH1 TH2 TH3 TH4
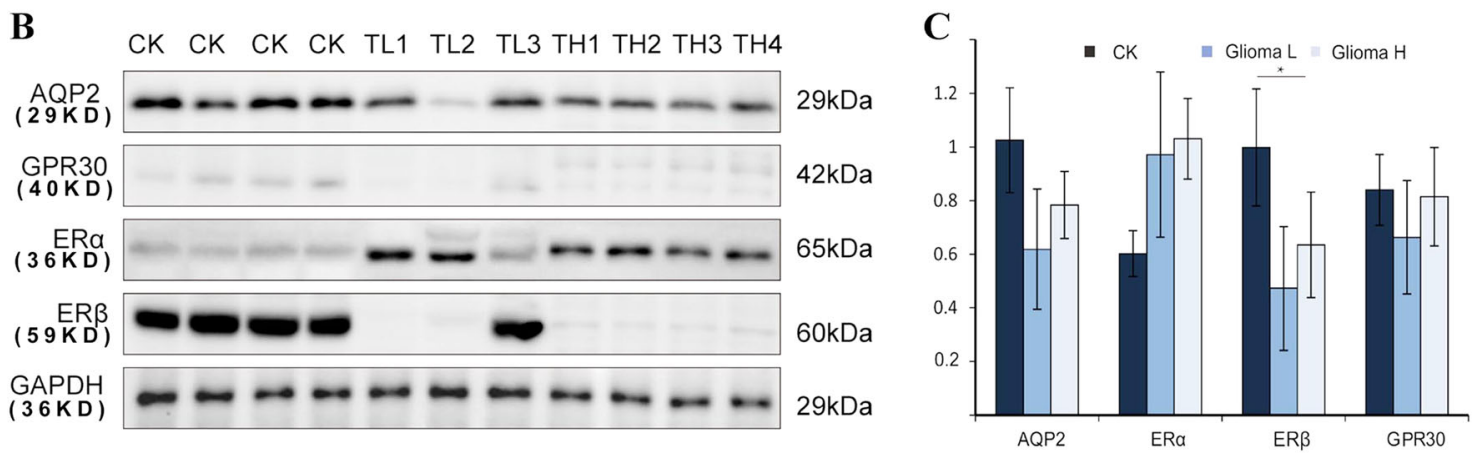

Fig. 1 Expression of AQP2 and ERs in glioma and glial tissues from patients. Fluorescence staining analysis showed that $A Q P 2, E R a$, and ERß were mainly located in the outer part of the nucleus (a). Target protein was stained in red. Cell nuclei were stained with DAPI. Western blot analysis demonstrated that the high expression levels of ERa, whereas low expression levels of ER $\beta$ and AQP2 were observed in glial cells compared to glioma cells $(\mathbf{b}, \mathbf{c}) .{ }^{* *} P<0.01$ compared with the corresponding control. CK normal tissues, T tumor group, TL low-grade tumor, TH high-grade tumor 
A

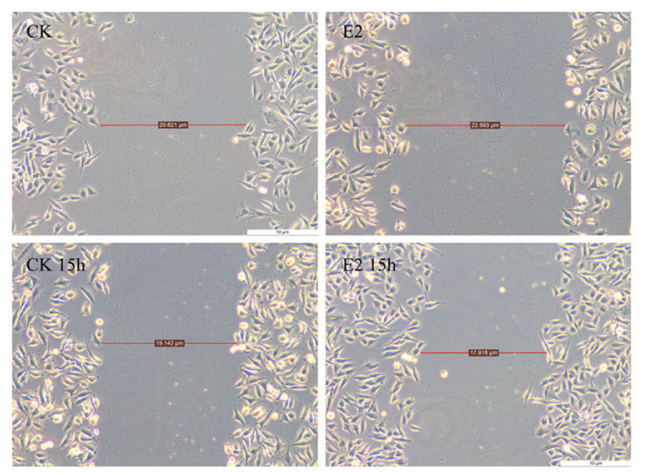

C

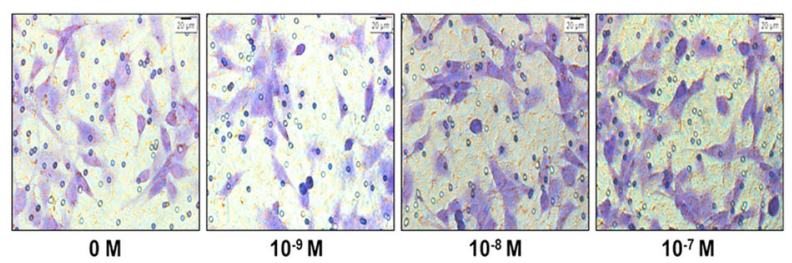

B

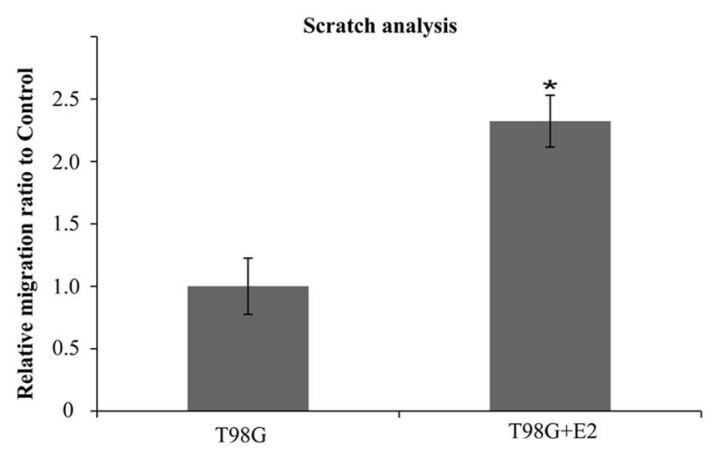

D

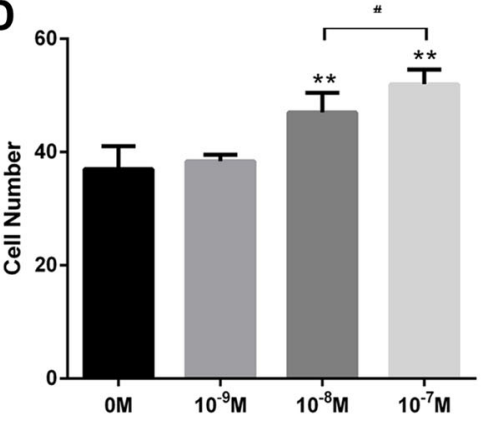

E

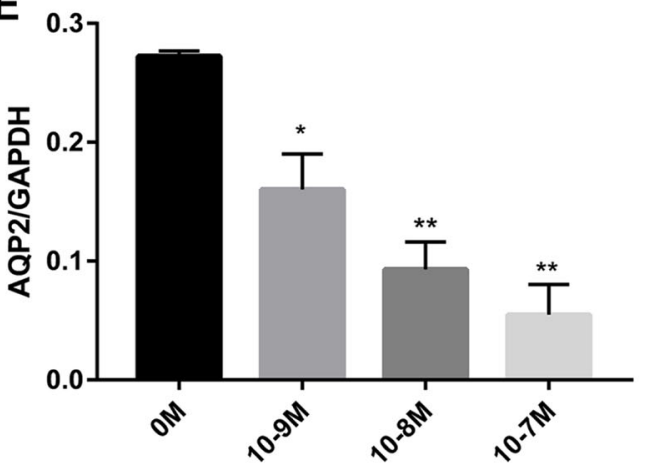

G

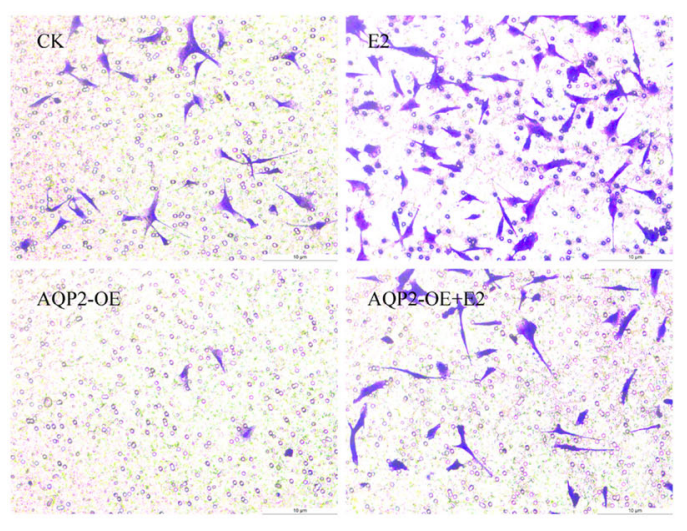

$\mathbf{F}$

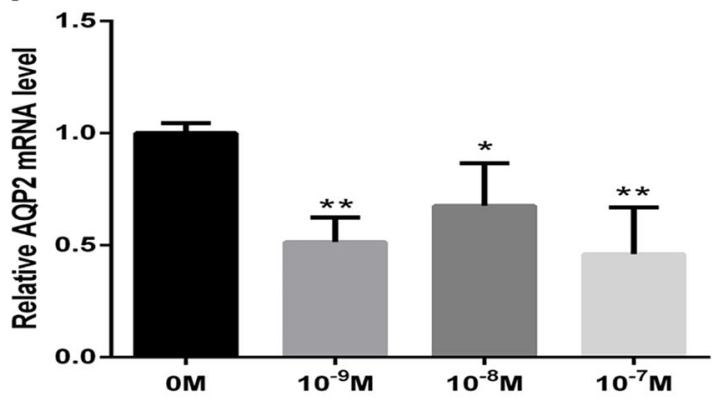

H

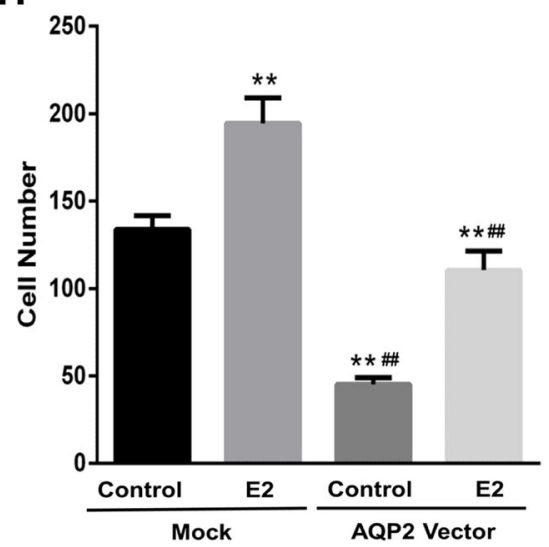

Fig. 2 Effects of E2 on cell migration and invasion in T98G and U87 cell lines. Estrogen (E2) significantly increased the T98G cell migration $(\mathbf{a}, \mathbf{b})$ and $\mathrm{U} 87$ cell invasion capacity $(\mathbf{c}, \mathbf{d})$ in a concentration-dependent manner, as tested by wound scratch assay and transwell assay, respectively. Both T98G and U87 are glioblastoma cell lines. e RT-qPCR showed that E2 decreased the mRNA levels, and (f) western blotting showed decreased protein levels of AQP2 compared to the control. Overexpression of AQP2 decreased cell invasion, which was attenuated by E2 ( $\mathbf{g}, \mathbf{h})$. Three independent experiments were repeated. ${ }^{*} P<0.01$ vs. control; ${ }^{*}{ }^{\#} P<0.05$ vs. control 
cell lines. The results demonstrated that AQP2 was located in the nucleus of the cell line; this finding was confirmed by two anti-AQP2 antibodies (Fig. 3a). This strange phenomenon was confirmed by six other malignant tumor cell lines (Fig. 3b). However, AQP2 was mainly expressed in the outer part of the nuclei in glial and glioma cells in the tissues (Fig. 1a). Based on GeneCards, AQP2 was supposed to locate mainly on the membrane. To further investigate the existence of a transmembrane mechanism of AQP2 in U87 cells, we performed a separation experiment of the cytoplasm and the nucleus. Under the treatment of $10^{-7} \mathrm{M}$ E2 for $48 \mathrm{~h}$, AQP2 expression was decreased both on the membrane and in the nucleus; however, there was no change observed in the cytoplasm (Fig. 3c, d). Thus, estrogen reduces the total amount of AQP2, rather than simply transferring AQP2 from the nucleus to the cell membrane. Since phosphorylated (p) AQP2 is the active form of $\mathrm{AQP2} 2^{24}$, does estrogen-induced cell migration be achieved by phosphorylation of AQP2 in the absence of a total AQP2? To study whether E2 decreases AQP2 accumulation on the membrane by inhibiting the phosphorylation of AQP2, the levels of $\mathrm{p}-\mathrm{AQP} 2$ in the nucleus and in the whole cell were measured. By western blot analysis, p-AQP2 (ser256), p-AQP2 (ser261), p-AQP2 (ser264), and p-AQP2 (thr269) were detected. E2 inhibited phosphorylation of total AQP2. Increased total amount of p-AQP2 (ser261), but decreased p-AQP2 (ser256) and p-AQP2 (ser264), was observed with E2, which were attenuated by overexpression of AQP2 (Fig. 3e, f). There were no obvious changes in p-AQP2 (ser264) and p-AQP2 (thr269) content both in total and in the nuclei (Fig. 3e-h). These data indicated that p-AQP2 (ser256) and p-AQP2 (ser264) of total AQP2 were consistent with the AQP2 quantity changes on the membrane. These findings indicated that E2 reduces P-AQP2 (ser256) or p-AQP2 (ser264) content on the membrane, which may inhibit the accumulation of AQP2 on the membrane and promote cell migration.

\section{E2 influences intranuclear AQP2 function by regulating AQP2 downstream genes}

Since the role of estrogen in AQP2 in the nucleus is not achieved by phosphorylation, is there a downstream gene for AQP2 in the nucleus that affects cell migration and is regulated by estrogen? To investigate AQP2-bound downstream genes in the nucleus, we performed chromatin immunoprecipitation sequencing (ChIP-seq) with anti-AQP2-flag, collected DNA, and performed sequencing. Gene Ontology analysis showed enrichment for biological processes, cellular components, and molecular functions. One hundred and thirty-eight genes were recruited. Three genes related to tumor metastasis, namely, ankyrin repeat and FYVE domain containing 1
(ANKFY1), lymphocyte transmembrane adaptor 1 (LAX1), and latent transforming growth factor beta-binding protein 1 (LTBP1) (provided by RefSeq, Apr 2012), were selected for follow-up studies to test the effects of AQP2 on these genes. Overexpression of AQP2 significantly upregulated the mRNA and protein levels of $L A X 1$, while E2 inhibited the regulatory effects of overexpression of AQP2 on $L A X 1$ (Fig. 4a-d). LAX1 was chosen for further study.

\section{ERs bound to AQP2 function as ANKFY1, LAX1, and $L T B P 1$ gene promoters}

To determine how E2 influences cell invasion by cooperating with intranuclear $\mathrm{AQP} 2$, the relationship between ERs, AQP2, and the downstream genes was investigated. U87 cells were transfected with the corresponding gene small interfering RNA (siRNA). The transwell assay results showed that, after treatment with ANKFY1siRNA, LAX1siRNA, and LTBP1siRNA, respectively, the cell invasion capacities were promoted compared to control lentivirus (Fig. $5 \mathrm{a}-\mathrm{f}$ ). The $L A X 1$ gene was selected as an example to investigate LAX1 expression via regulation of $\mathrm{AQP} 2$ at the transcriptional level. After transfection with AQP2 + pGL3-LAX1 successfully (Fig. 5g), our results showed that overexpression of AQP2 increased LAX1 expression, while LAX1siRNA decreased AQP2 effects on LAX1 expression (Fig. 5h). AQ2 vector decreased cell invasion, while it was reversed by LAX1siRNA (Fig. 5c). Overexpression of ER $\beta$ upregulated the mRNA levels of ANKFY, LAX, LTBP, and AQP2, while ER $\alpha s i R N A$ increased the mRNA levels of ANKFY, LAX, LTBP, and AQP2 compared to those of the control groups (Fig. 5 j, k). These data indicated that ER $\alpha$ and ER $\beta$ play an inverse influence on AQP2.

\section{Discussion}

In this regard, the present study provided the following novel findings: (1) AQP2 expression was decreased in glioma cells in tissues; (2) AQP2 was located in all parts of glioma cells or glial cells in the tissues and was located mainly in the nuclei in cell lines; (3) E2 decreased AQP2 expression in cell lines, particularly in the nucleus; (4) E2 promoted cell invasion by reducing AQP2 distribution in the nucleus; and (5) the plausible molecular mechanisms by which E2 regulates AQP2 sublocalization and promotes cell invasion occurrence at the nuclear and membrane levels. E2 inhibited the phosphorylation of AQP2 of the membrane, which may reduce AQP2 accumulation on the membrane. At the nuclear level, AQP2-bound ER $\alpha$ / ER $\beta$ functioned as the promoters of ANKFY1, LAX1, and $\angle T B P 1$ genes.

The role of estrogen in glioma development remains controversial. Estrogens can exert their effects through intracellular or membrane-associated ERs, such as the 


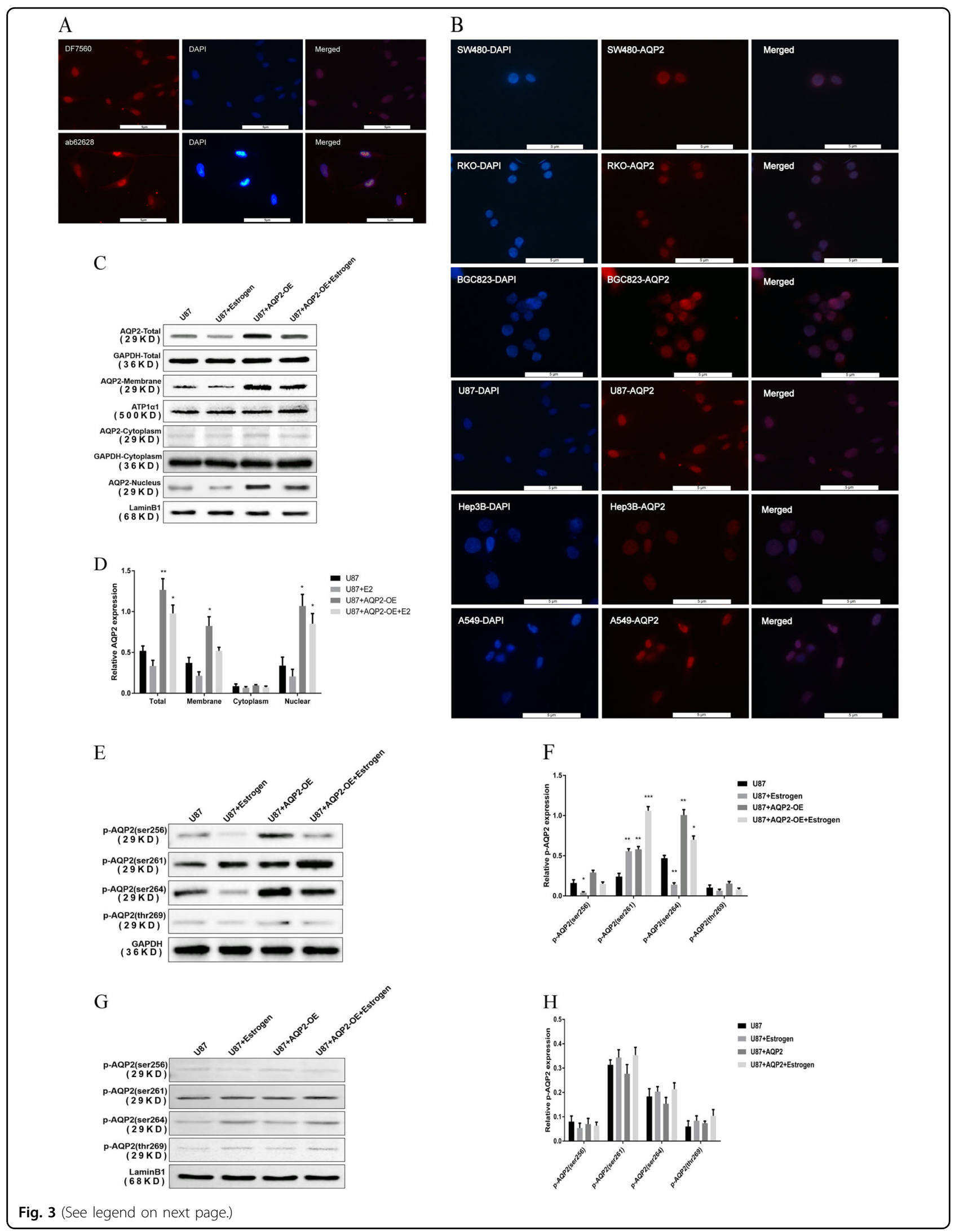


(see figure on previous page)

Fig. 3 Effects of E2 on intranuclear AQP2 expression in tumor cell lines. a Experiments were repeated with two antibodies of AQP2 (DF7569 and Ab62628) in U87 cells. AQP2 was stained in red. Cell nuclei were stained with DAPI. $\mathbf{b}$ The phenomena of AQP2 sublocalization in the nuclei in cell lines were repeated and were confirmed in six other cell lines. c, $\mathbf{d}$ Western blot analysis of the cytoplasm and nucleus separately showed that E2 decreased total AQP2 expression in both the nucleus and cell membranes without change in the cytoplasm of U87 cells. $\mathbf{c}$, $\mathbf{d}$ showed the results of RT-qPCR and western blotting, respectively. e, f E2 inhibited the total expression of p-AQP2 (ser256) and p-AQP2 (ser264) tested by western blotting and RT-qPCR, respectively. High expression level of both p-AQP2 (ser256) and p-AQP2 (ser264) observed in the AQP2 overexpression was attenuated by E2. The total expression level of P-AQP2 (ser261) was promoted by E2 and attenuated by overexpression of AQP2. ${ }^{* *} P<0.001$ compared to control. $\mathbf{g}$, $\mathbf{h}$ There was no obvious phosphorylation observed in the nucleus tested by western blotting and RT-qPCR, respectively. Three independent experiments were repeated. ${ }^{* *} P<0.01$ compared to control, ${ }^{*} P<0.05$ vs. control. AQP2-membrane cell membrane, OE overexpression
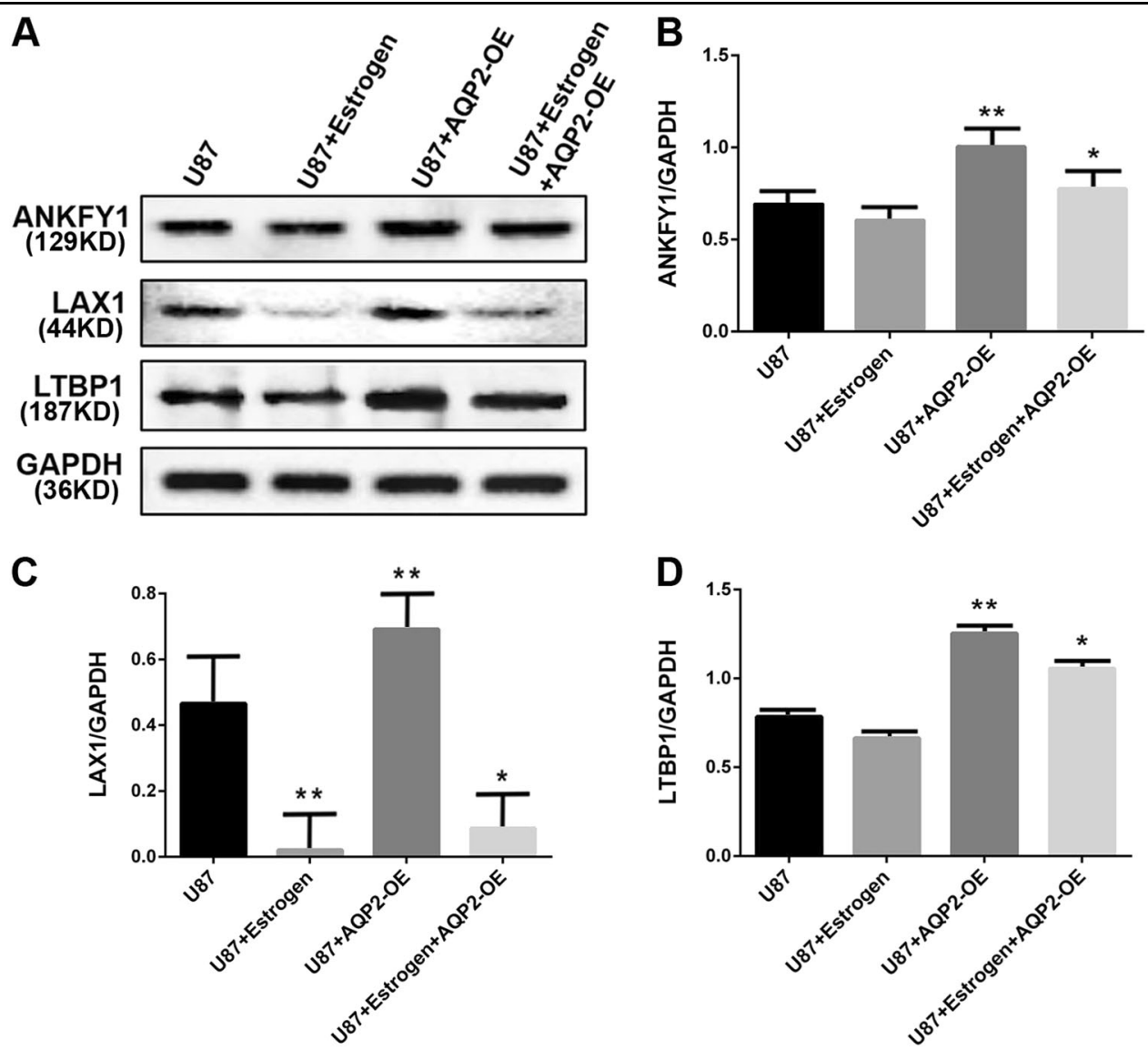

Fig. 4 E2 influences AQP2 in the U87 cell nucleus regulated by ANKFY1, LAX1, and LTBP1 genes. The expression of ANKFY1, LAX1, and LTBP1 in U87 cell lines was analyzed by western blot (a) and RT-qPCR (b-d). E2 decreased the expressions of ANKFY1, LTBP1, and LAX1 genes in nucleus of U87 cell line. Overexpression of AQP2 promoted the expressions of ANKFY1, LAX1, and LTBP1 genes, whereas it was attenuated by E2. Three independent experiments were repeated. ${ }^{*} P<0.01$ vs. control, ${ }^{*} P<0.05$ vs. control. NC normal cells, AQP2NCsiRNA overexpression of AQP2

intracellular receptors $E R \alpha / E R \beta$ and GPRs. In this study, $E R \alpha$ protein expression levels were higher in glioma cells than in glial cells, while ER $\beta$ levels were significantly decreased in high-grade glioma compared with normal glial cells. This result was consistent with other reports that suggested that high expression of ER $\beta$ was an independent, favorable prognostic factor, but ER $\alpha$ was a poor prognostic factor in the multivariate analysis ${ }^{25,26}$. In this study, there was no significant difference in GPR30 expression between glioma cells and glial cells in the tissues. In addition to neurons and astrocytes, other cells, such as microglia and macrophage-like members of the intrinsic brain immune system, also express nuclear and nonnuclear ERs ${ }^{27}$. Experimental studies have shown that ER $\beta$ inhibits the proliferation of gliomas and induces cell death $^{28}$. ER $\beta$-selective agonists were found to inhibit the proliferation of glioma cell lines in vitro ${ }^{29}$. Thus, we inferred that the receptor quantity or ratio in astrocytic 


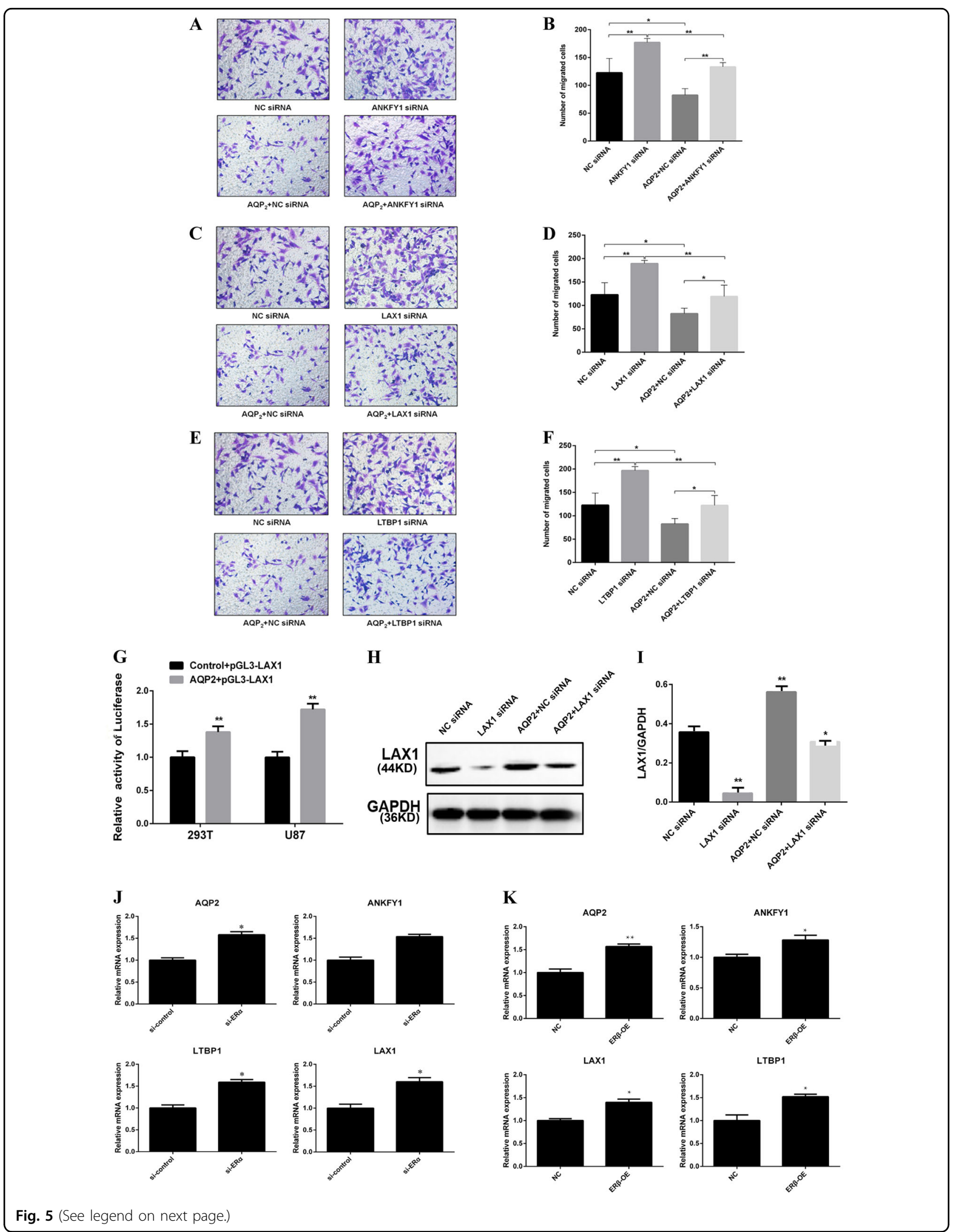


(see figure on previous page)

Fig. 5 The pathway of E2 influences the localization of AQP2 in the U87 cell nucleus. Invasion of U87 cell was influenced by siRNA in relation to ANKFY1, LAX1, and LETP1 genes analyzed using the transwell assay (a-f). Overexpression of AQP2 decreased the cell invasion, while it was attenuated by siRNA in relation to ANKFY1, LAX1, and LETP1 genes. g showed that AQP2 + PGL3-LAX1 was loaded using HEK 293T vectors and transfected successfully to the U87 cell line. Luciferase reporter assays were performed. $\mathbf{h}$, i Western blot and RT-qPCR showed LAX1 gene expression in the nucleus. AQP2 promoted LAX1 expression, which was attenuated by LAX1siRNA. $\mathbf{j}$ showed that siRNA ERa increased ANKFY1, LAX1, LETP1, and AQP2 mRNA levels and was further corroborated by the overexpression of ER $\beta$ condition analyzed by RT-qPCR $(\mathbf{k})$. The results are expressed as the means \pm SEM of three independent experiments. ${ }^{*} P<0.05,{ }^{*} P<0.01$ vs. control. Si siRNA, AQP2NCsiRNA overexpression of AQP2

cells may influence E2 function and the prognosis of gliomas.

The underlying mechanisms of the regulation of AQP transcription via estrogen are complex. AQP2 forms a water-specific channel that provides the plasma membranes of renal collecting ducts with a high water permeability, thereby permitting water to move into the cells in the direction of an osmotic gradient. There have been no reports regarding AQP2 expression in gliomas. An important paralog of this gene is AQP5. It is known that phosphorylation of AQP5 results in internalization of the protein from the plasma membrane ${ }^{30}$. AQP5 showed dramatic adaptation to a changed environment and translocates into the nucleus by in vitro culture ${ }^{31}$. This is the precedent of the discovery of AQP2 with differential sublocalization in gliomas, with or without pretreatment with E2. Overexpression of AQP2 in the nuclei of U87 cells reduced cell invasion, suggesting the involvement of regulatory migration genes in this process. Upon binding of estrogen to an ER, the ligand receptor complex dimerizes and migrates into the nucleus, where the dimer binds to hormone response elements (HREs) in the promotor region of estrogen-responsive genes. Activation of the HRE leads to the induction or repression of gene transcription. Our ChIP sequence and luciferase reporter system indicated that AQP2-bound ER $\alpha / E R \beta$ functioned as a promoter of $A N K F Y 1, L A X 1$, and LTBP1 genes in the nucleus. In our previous study, we proved that there were EREs in the AQP2 promoter $^{18}$. This finding supported that AQP2 might bind to ER $\alpha / E R \beta$ and function as a promoter of ANKFY1, LAX1, and LTBP1 genes in the nucleus. Furthermore, AQP2 promoted the transcription and expression of ANKFY1, LAX1, and LTBP1 genes.

Phosphorylated AQP2 on the membrane provides a necessary and sufficient condition for the role of AQP2, while phosphorylation is not required for its function in the nucleus. According to the STRING database and group-based prediction system, AQP2 binds to protein kinase, cAMP-dependent, catalytic beta (PRKACB), directly, which was triggered by receptor binding to Gprotein-coupled receptors. Protein kinase A activation regulates intracellular transport mechanisms and ion flux $^{32}$. Moreover, GPR30 is one of the receptors of estrogen. Our findings showed that GPR30 was highly expressed on the membranes of glioma cells. E2 may bind to GPR30 and trigger PRKACB. This may inhibit phosphorylation of AQP2 also. In the present study, after E2 treatment, the changing trends in the total protein of $\mathrm{p}$ AQP2 (ser256) and p-AQP2 (ser264) were consistent with the changing trend of AQP2 content on the cell membrane. As described in previous reports, there are reports of p-AQP2 (ser256), p-AQP2 (ser264), and AQP2 accumulation on the cell membrane ${ }^{33}$. Therefore, it is likely that E2 might reduce the accumulation of AQP2 on the membrane by lowering the content of p-AQP2. Thus, we concluded that E2 may reduce AQP2 accumulation on the membrane by inhibiting phosphorylation of AQP2 and reducing the quantity of $\mathrm{AQP} 2$ in the nucleus.

In short, our findings successfully proved that AQP2 regulation of glioma cell invasion was induced by E2. A novel transmembrane phenomenon in glioma cells was discovered. We proposed that E2 may reduce AQP2 accumulation on the membrane by inhibiting phosphorylation of AQP2 on the membrane and reducing the quantity and sublocalization of AQP2 in the nucleus.

\section{Materials and methods \\ Subjects and sample collection}

Ethical approval for this project was granted by the Ethics Committee of the First Hospital affiliated to the School of Medicine, Zhejiang University (No. 2018-500). Written informed consent was obtained from each subject before tissue collection. The glial samples were collected from patients $(n=4$, two women and two men, median age $=47$ years; range, $40-55$ years) who attended to the Department of Neurosurgery of the First Hospital Affiliated of School of Medicine, Zhejiang University for brain injuries and were obtained by biopsy for diagnostic purposes. The glioma samples were collected from patients $(n=4$, two women and two men; median age, 50 years; range, 44-67 years) who underwent surgical treatment for glioma (International Federation of Gynecology and Obstetrics (FIGO)) stage III and IV patients $(n=3$, two women and one man; median age, 46 years; range, 43-48 years) and who underwent surgical treatment for glioma (FIGO) stage I and II. None of these patients received preoperative radiation or chemotherapy. Diagnosis of glioma was pathologically confirmed. No patients had received hormone therapy in the prior 3 months. 
Table 1 Sequences of the primers used for real-time PCR

\begin{tabular}{|c|c|c|}
\hline Gene & Forward $\left(5^{\prime}-3^{\prime}\right)$ & Reverse $\left(5^{\prime}-3^{\prime}\right)$ \\
\hline$A Q P 2$ & CGTTTGGCTTGGGTATTGGC & CGGATGTCTGCTGGCGTGA \\
\hline AQP4 & GAGTGACAGACCCACAGCAAGG & AAAGCAAAGGGAGATGAGAACC \\
\hline AQP5 & GGCTGCCATCCTTTACTTCTACCT & GCTCCTCCCAGTCCTCGTCA \\
\hline$A Q P 8$ & CGTTCTCCATCGGCTTTGC & AGGCGGGTCTTCCCATCTC \\
\hline ANKFY1 & TAGCACCATTTGAAATCAGTGTT & TGGTGTCGTGGAGTCG \\
\hline$L A X 1$ & CGCAAGGATGACACGCAAATTC & TGGTGTCGTGGAGTCG \\
\hline$\angle T B P 1$ & GTCCTGGTGGAATGGGTTATAC & GTTGAGTGTTCTTTGGCTTGAC \\
\hline LARP1 & GTTTCCTACCCATCACCCTTATT & CACCACCTTGCTGTCCTTTA \\
\hline RFC3 & GGCTCATAGACTTGCAGAGAAG & CCCAATCTGTCTCAGGGATTTC \\
\hline SIRNA NC & UUCUCCGAACGUGUCACGUTT & ACGUGACACGUUCGGAGAATT \\
\hline ANKFY1 siRNA1 & GCUGCAGUGCAAACAACUATT & UAGUUGUUUGCACUGCAGCTT \\
\hline ANKFY1 SIRNA2 & GGACUUCAUUUGAUGAGAATT & UUCUCAUCAAAUGAAGUCCTT \\
\hline ANKFY1 siRNA3 & GCCCAUGUCAACCACAGAATT & UUCUGUGGUUGACAUGGGCTT \\
\hline LAX1 SiRNA1 & GGAAUUGGAAUAAACGGAATT & UUCCGUUUAUUCCAAUUCCTT \\
\hline LAX1 SiRNA2 & GCAUACAGCCCACAUCCAUTT & AUGGAUGUGGGCUGUAUGCTT \\
\hline LAX1 SiRNA3 & GCUGAGACUCUAGCUUCUATT & UAGAAGCUAGAGUCUCAGCTT \\
\hline LTBP1 siRNA1 & GCCAUCUUCCAUGUAUGAATT & UUCAUACAUGGAAGAUGGCTT \\
\hline LTBP1 SIRNA2 & GCCUAAACUUUAUCAGCAUTT & AUGCUGAUAAAGUUUAGGCTT \\
\hline LTBP1 SIRNA3 & GCCAAUCCCAAGUCUCGUATT & UACGAGACUUGGGAUUGGCTT \\
\hline ESR1shRNA & GAGGGAGAAUGUUGAAACATT & UGUUUCAACAUUCUCCCUCTT \\
\hline ESR1shRNA & GGUUCCGCAUGAUGAAUCUTT & AGAUUCAUCAUGCGGAACCTT \\
\hline ESR1shRNA & GGCUAGAGAUCCUGAUGAUTT & AUCAUCAGGAUCUCUAGCCTT \\
\hline GAPDH & ACTCCCATTCTTCCACCTTTG & CCCTGTTGCTGTAGCCATATT \\
\hline
\end{tabular}

\section{Cell culture condition}

The U87 cell line, derived from male glioblastomas, was obtained from the American Type Culture Collection (Manassas, VA, USA, ATCC HTB-14, USA). The T98G cell line, derived from male glioblastoma multiforme, was obtained from China Shanghai Zeye Biological Technology Co., Ltd. The p3B2.1-7 cell line, derived from human liver cancers; the RKO cell line, derived from human colonic cancer; the A549 cell line, derived from human non-small-cell lung cancer; the SW480, derived from human colonic cancer; and the BGC823, derived from human gastric cancer were all obtained from the Chinese Academy of Sciences, Shanghai Life Science, Cell Resource Center. The cell lines were cultured as previously described ${ }^{34}$.

\section{RNA extraction and qRT-PCR}

Total RNA was extracted and purified using the Universal RNA Extraction Kit (MiniBEST, TaKaRa, Japan) according to the manufacturer's instructions. Real-time PCR amplifications were conducted in triplicate. The primer sequences used for quantitative real-time PCR (qRT-PCR) are listed in Table 1.

\section{Western blot analysis}

For western blotting, cells were lysed in RIPA buffer (Beijing Solarbio Science and Technology Co., Ltd., Beijing, China).

\section{Transwell migration assay}

Cell culture inserts were seeded with $5 \times 10^{5}$ of the cell lines. The U87 and T98G cells were treated with different concentrations $\left(10^{-9}, 10^{-8}\right.$, or $\left.10^{-7} \mathrm{M}\right)$ of E2 (SigmaAldrich, E8875). Cells on the lower aspect of the mounted membranes were viewed and photographed under a microscope every $24 \mathrm{~h}$. For quantification, the number of stained cells in five selected areas (top, middle, bottom, left, and right) was manually counted. 


\section{Wound scratch assay}

Different groups of cells were grown in serum-free culture to confluence in a six-well plate. After $12 \mathrm{~h}$, the cells were wounded, and the healing capability was calculated by evaluating the amount of cell coverage to the scratch zone at 0 and $24 \mathrm{~h}$.

\section{Fluorescent staining}

Cells were blocked with Dako Protein Block (Dako, Mississauga, ON, Canada) as previously described ${ }^{18}$. The AQP2 antibody ( $1: 100$ diluted in Dako Protein Block, DF7560, Affinity Co., Cincinnati, OH, USA), the corresponding secondary donkey anti-rabbit IgG H\&L (1:100 diluted in Dako Protein Block, Alexa Fluor 647), another AQP2 antibody (1:500 diluted in Dako Protein Block, Ab62628, Abcam Co., USA), the corresponding secondary goat anti-rabbit IgG H\&L (1:10,000 diluted in Dako Protein Block, BL003A, Biosharp), anti-ER $\alpha$ (1:100 diluted in Dako Protein Block, Proteintech, 21244.1.AP), rabbit antiER $\beta$ mouse (1:100 diluted in Dako Protein Block, Santa Cruz Sc-53494), and horseradish peroxidase-conjugated secondary antibodies (MultiSciences GAR007, GAM007) were used. Finally, the cells were counterstained with the chromosomal dye 4',6-diamidino-2-phenylindole (DAPI) (Beyotime Biotechnology, C1006).

\section{Separation of the nucleus and cytoplasm}

According to the manufacturer's instructions (Membrane Nuclear and Cytoplasmic Protein Extraction Kit, Sangon Biotech, China; C510002), protein from the nucleus, cytoplasm, and membrane was obtained.

\section{RNA interference experiments}

ANKFY1, LAX1, LTBP1, and ER- $\alpha$ (ESR1) siRNA duplexes were chemically synthesized by Ambion (Carlsbad, CA, USA). Silencer FAM-labeled negative siRNA was used as a control oligonucleotide. The primer sequences used for the qRT-PCR are listed in Table 1.

\section{Lentiviral vector construction and transfection}

Lentivirus vectors expressing flag-tagged AQP2 and ER $\beta$ (HG11302-ACG) were generated by inserting the corresponding complementary DNA into the multicloning site of the lentivirus backbone plasmid of pSLLVCMV-Zsgreen-puro and Pcmv3-C-GFPSpark, respectively. The constructs were co-transfected with packaging vectors into $293 \mathrm{~T}$ cells for packaging, which was followed by purification (Cell-Land Biological Technology, Hangzhou, China).

\section{Bioinformatics analysis and ChIP}

The primer sequences used to amplify the precipitated DNA are listed in Table 1. Target gene sequences were constructed from the NCBI databases (http://www.ncbi. nlm.gov/gene). The pCDH-CMV-MCS-EF1-copGFPAQP2 vector with the AQP2-flag fusion protein was constructed, and the AQP2 lentivirus packaging was successful and highly infected. ChIP-seq was performed as previously described ${ }^{35}$.

\section{Plasmid construction and luciferase reporter assay}

To predict AQP2 binding to the DNA of the LAX1 promoter at conserved sites, we constructed a luciferase reporter plasmid with the LAX1 promoter ligated to the luciferase expression vector pGL3. DNA fragments of human LAX1 promoters were amplified from NCBI after being compared with the original LAX1 sequence, and the vector was constructed successfully. The luciferase values were normalized to the $293 \mathrm{~T}+$ pGL3-Basic or U87 + pGL3-Basic vector.

\section{Statistics}

Multiple comparisons were analyzed by one-way or two-way analysis of variance, followed by multiple comparison tests using the SPSS 19.0 software (SPSS, Chicago, IL, USA). Statistical significance was defined as $P<0.05$.

\section{Acknowledgements \\ This work was supported by the National Natural Science Foundation of Zhejiang Province (Nos. LY17H040003 and Y18H040008). \\ Author details \\ ${ }^{1}$ Department of Neurosurgery, The First Affiliated Hospital, Zhejiang University School of Medicine, Hangzhou Shi, Zhejiang Province, China. ${ }^{2}$ Department of Otolaryngology, Hangzhou Children's Hospital, Hangzhou City, Zhejiang Province, China. ${ }^{3}$ Key Laboratory of Head and Neck Cancer Translational Research, Department of Head and Neck Surgery of Zhejiang Cancer Hospital, Hangzhou City, Zhejiang Province, China. ${ }^{4}$ Department of Gynecology, Womens Hospital, Zhejiang University School of Medicine, Hangzhou City, Zhejiang Province, China. ${ }^{5}$ Cell-land Biology Technology Co., Ltd., Hangzhou City, Zhejiang Province, China}

Conflict of interest

The authors declare that they have no conflict of interest

\section{Publisher's note}

Springer Nature remains neutral with regard to jurisdictional claims in published maps and institutional affiliations.

The online version of this article (https://doi.org/10.1038/s41420-018-0113-y) contains supplementary material, which is available to authorized users.

Received: 4 August 2018 Revised: 18 September 2018 Accepted: 26 September 2018

Published online: 17 October 2018

\footnotetext{
References

1. Qi, Z. Y. et al. Exogenous and endogenous hormones in relation to glioma in women: a meta-analysis of 11 case-control studies. PLOS ONE 8, e68695 (2013).

2. Chakrabarti, M. et al. Estrogen receptor agonists for attenuation of neuroinflammation and neurodegeneration. Brain Res Bull. 109, 22-31 (2014).

3. Allen, N. J. \& Barres, B. A. Neuroscience: glia-more than just brain glue. Nature 457, 675-677 (2009).
} 
4. Bao, D. et al. Regulation of p53wt glioma cell proliferation by androgen receptor-mediated inhibiton of small VCP/p97-ineracting protein expression. Oncotarget 8, 23142-23154 (2017).

5. Gutiérrez, R. A., Hansberg, P. V. \& Camacho, A. I. Proliferative and invasive effects of progesterone-induced blocking factor in human glioblastoma cells. Biomed. Res. Int. 2017, 1295087 (2017).

6. Cookman, C. J. \& Belcher, S. M. Estrogen receptor- $\beta$ up-regulates IGF1R expression and activity to inhibit apoptosis and increase growth of medulloblastoma. Endocrinology 156, 2395-408 (2015).

7. Batistatou, A. et al. Estrogen receptor beta (ERbeta) is expressed in brain astrocytic tumors and declines with dedifferentiation of the neoplasm. J. Cancer Res. Clin. Oncol. 130, 405-410 (2004).

8. Jover, M. T. et al. Molecular mechanisms mediating the neuroprotective role of the selective estrogen receptor modulator, bazedoxifene, in acute ischemic stroke: a comparative study with 17ß-estradiol. J. Steroid Biochem. Mol. Biol. 171, 296-304 (2017)

9. Verkman, A. S., Yang, B., Song, Y., Manley, G. T. \& Ma, T. Role of water channels in fluid transport studied by phenotype analysis of aquaporin knockout mice. Exp. Physiol. 85(Suppl. 1), 233S-241S (2000).

10. Verkman, A. S., Hara-Chikuma, M. \& Papadopoulos, M. C. Aquaporins-new players in cancer biology. J. Mol. Med. 86, 523-529 (2008).

11. Papadopoulos, M. C., Saadoun, S. \& Verkman, A. S. Aquaporins and cell migration. Pflug. Arch. 456, 693-700 (2008).

12. Saadoun, S., Papadopoulos, M. C., Hara-Chikuma, M. \& Verkman, A. S. Impairment of angiogenesis and cell migration by targeted aquaporin-1 gene disruption. Nature 434, 786-92 (2005).

13. Verkman, A. S. More than just water channels: unexpected cellular roles of aquaporins. J. Cell Sci. 118, 3225-32 (2005).

14. Hildenbrand, A. et al. Aquaporin 1 is expressed in the human endometrium during normal cycle and increases after mifepristone treatment. Int. J. Mol. Med. 22, 49-53 (2008).

15. Saadoun, S., Papadopoulos, M. C., Davies, D. C., Krishna, S. \& Bell, B. A. Aquaporin-4 expression is increased in oedematous human brain tumours. J. Neurol. Neurosurg. Psychiatry 72, 262-5 (2002).

16. Warth, A. et al. Expression pattern of the water channel aquaporin-4 in human gliomas is associated with blood-brain barrier disturbance but not with patient survival. J. Neurosci. Res. 85, 1336-46 (2007).

17. Huang, H. F. et al. Function of aquaporins in female and male reproductive systems. Hum. Reprod. Update 12, 785-95 (2006).

18. Zou, L. B. et al. Identification of estrogen response element in the aquaporin-2 gene that mediates estrogen-induced cell migration and invasion in human endometrial carcinoma. J. Clin. Endocrinol. Metab. 96, E1399-408 (2011).

19. Fushimi, K. et al. Cloning and expression of apical membrane water channel of rat kidney collecting tubule. Nature 361, 549-552 (1993).
20. Jiang, X. X. et al. Immunohistochemical detection of aquaporin expression in eutopic and ectopic endometria from women with endometriomas. Fertil. Steril. 94, 1229-1234 (2010).

21. Jiang, X. X. et al. Aquaporin 5 plays a role in estrogen-induced ectopic implantation of endometrial stromal cells in endometriosis. PLOS ONE 10 e0145290 (2015).

22. Dueñas Jiménez, J. M. et al. Aromatase and estrogen receptor alpha mRNA expression as prognostic biomarkers in patients with astrocytomas. J. Neurooncol. 119, 275-84 (2014).

23. Liu, J. et al. Differential effects of estrogen receptor $\beta$ isoforms on glioblastoma progression. Cancer Res. 78, 3176-3189 (2018).

24. Valenti, G. et al. The phosphatase inhibitor okadaic acid induces AQP2 translocation independently from AQP2 phosphorylation in renal collecting duct cells. J. Cell Sci. 113, 321-336 (2000).

25. Habib, P. \& Beyer, C. Regulation of brain microglia by female gonadal steroids. J. Steroid Biochem. Mol. Biol. 146, 3-14 (2015).

26. Vegeto, $E$. et al. Estrogen receptor-alpha mediates the brain antiinflammatory activity of estradiol. Proc. Natl. Acad. Sci. USA 100, 9614-9619 (2003).

27. $\mathrm{Wu}, \mathrm{W}$. F. et al. Targeting estrogen receptor beta in microglia and T cells to treat experimental autoimmune encephalomyelitis. Proc. Natl. Acad. Sci. USA 110, 3543-3548 (2013).

28. Paterni, I. et al. Highly selective salicylketoxime-based estrogen receptor $\beta$ agonists display antiproliferative activities in a glioma model. J. Med. Chem. 58, 1184-1194 (2015)

29. Sartorius, C. A. et al. Estrogen promotes the brain metastatic colonization of triple negative breast cancer cells via an astrocyte-mediated paracrine mechanism. Oncogene 35, 2881-2892 (2016).

30. Kumari, S. S. et al. Sptial expression of aquaporin 5 in mammalian cornea and lens and regulation of its localization by phosphokinase A. Mol. Vis. 18 957-967 (2012)

31. Jae, W. P., Yun, K. S. \& Yong, P. C. Adaptive transition of aquaporin 5 expression and localization during preimplantation embryo development by in vitro culture. Dev. Reprod. 18, 153-160 (2014).

32. Isobe, $\mathrm{K}$. et al. Systems-level identification of PKA-dependent signaling in epithelial cells. Proc. Natl. Acad. Sci. USA 114, E8875-E8884 (2017).

33. Hua, J. L. et al. The phosphorylation state of serine 256 is dominant over that of serine 261 in the regulation of AQP2 trafficking in renal epithelial cells. Am J Physiol Ren. Physiol. 295, F290-F294 (2009).

34. Ruan, H. et al. A novel peptide ligand RAP12 of LRP1 for glioma targeted drug delivery. J. Control Rel. 279, 306-315 (2018).

35. Kim, T. H. \& Dekker, J. ChIP-chip. Cold Spring Harb. Protoc. 5, pdb.prot 082636 (2018). 